\title{
The Genome of Shope Fibroma Virus, a Tumorigenic Poxvirus, Contains a Growth Factor Gene with Sequence Similarity to Those Encoding Epidermal Growth Factor and Transforming Growth Factor Alpha
}

\author{
WEN CHANG, ${ }^{1,2}$ CHRIS UPTON, ${ }^{2}$ SHIU-LOK HU, ${ }^{2}$ A. F. PURCHIO, ${ }^{2}$ AND GRANT MCFADDEN ${ }^{3 *}$ \\ Department of Microbiology and Immunology, University of Washington, Seattle, Washington 981951; Oncogen, Seattle, \\ Washington 98121 USA $^{2}$; and Department of Biochemistry, University of Alberta, Edmonton, Alberta, Canada T6G $2 \mathrm{H}^{3}$
}

Received 14 July 1986/Accepted 19 September 1986

\begin{abstract}
Degenerate oligonucleotide probes corresponding to a highly conserved region common to epidermal growth factor, transforming growth factor alpha, and vaccinia growth factor were used to identify a novel growth factor gene in the Shope fibroma virus genome. Sequence analysis indicates that the Shope fibroma growth factor is a distinct new member of this family of growth factors.
\end{abstract}

Poxviruses are distinguished from other eucaryotic DNA viruses in that all stages of viral replication occur in virosomes or micronuclei in the cytoplasm of infected cells (reviewed in references 9 and 18). Despite the fact that members of this virus group do not physically enter the cell nucleus during their replicative cycle, certain poxviruses have been recognized for many years as causative agents for a number of proliferative diseases. Three notable examples of such tumorigenic poxviruses are (i) Shope fibroma virus (SFV), which induces benign fibromas in adult rabbits (22) and invasive atypical fibrosarcomas in both newborn rabbits $(1,21,23,26)$ and immunosuppressed adult rabbits $(2,23)$; (ii) Yaba tumor virus, found to cause subcutaneous histiocytomas in monkeys and humans (3); and (iii) molluscum contagiosum virus, which causes benign tumorlike epidermal lesions in humans (7). Most of what little is known about the proliferative response induced by some members of the poxvirus family has come from classical biological studies of SFV-infected rabbits (reviewed in reference 11). At present it is unclear how, at the molecular level, SFV induces proliferation of target fibroblasts or the viral gene products which presumably mediate this response. However, it has been shown recently that vaccinia virus, a cytolytic poxvirus of the genus Orthopoxvirus, encodes a gene product designated vaccinia growth factor (VGF), which shares amino acid homology with epidermal growth factor (EGF) and transforming growth factor alpha $(\mathrm{TGF} \alpha)(5,6,19)$. These polypeptide growth factors bind to the EGF receptor on the cell surface and lead to the phosphorylation of the receptor in a process which eventually triggers cellular proliferation (reviewed in references $12,13,15,17$, and 20). Here we demonstrate that SFV also encodes a related gene product which possesses significant homology with the EGF family of growth factors.

To detect the growth factor gene of SFV, we used degenerate oligonucleotides as probes in hybridization studies. A region of seven amino acids (a.a.'s 75 to 81) in VGF was chosen for the design of the oligonucleotide probes (Fig. 1). The a.a. sequences in this region constitute a part of the cysteine loop which is highly conserved among the EGF

\footnotetext{
* Corresponding author.
}

family of growth factors. A pool of oligonucleotides (YC-1) was synthesized by using an Applied Biosystems 380A synthesizer, according to the preferred codon usage of vaccinia virus, to include 128 -fold degeneracy (as described in legend to Fig. 1) in the nucleotide sequences encoding VGF in this region. Using a ${ }^{32} \mathrm{P}$-end-labeled $\mathrm{YC}-1$ probe, we screened the cloned BamHI library of the SFV genome (30) by dot blot analysis, under washing conditions of $6 \times$ SSC ( $1 \times \mathrm{SSC}$ is $0.15 \mathrm{M} \mathrm{NaCl}$ plus $0.015 \mathrm{M}$ sodium citrate [pH 7]) at $37^{\circ}$ for $1 \mathrm{~h}$, and found that the BamHI C fragment contained sequences homologous to the growth factor family (Fig. 2i). The other faint signals (Fig. 2iA) were not reproducibly observed and represent background signals of

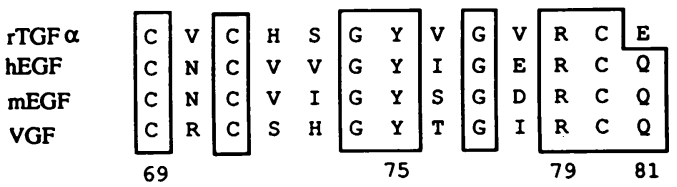

$$
\begin{aligned}
& \text { Codon degeneracy : } \\
& \text { 5. TAtacaggataAgatgccaA } 3 \text { ' }
\end{aligned}
$$

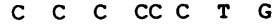

$$
\begin{aligned}
& \begin{array}{llll}
G & G & T & G
\end{array} \\
& \text { 3. atatgaccatatctacagtC } 5 \text { ' } \\
& \text { G } \begin{array}{ccc}
\text { C } & \mathbf{C} & \mathbf{C} \\
\mathbf{G} & \mathbf{G} & \mathbf{G}
\end{array} \\
& \begin{array}{lll}
\mathbf{T} & \mathbf{T} & \mathbf{T}
\end{array}
\end{aligned}
$$

FIG. 1. Oligonucleotide probe for the detection of the growth factor-related gene in SFV. A partial amino acid sequence of VGF (a.a.'s 69 to 81) was aligned with its counterparts in human and mouse EGF and in rat TGF $\alpha$. The highly conserved VGF region YTGIRCQ (a.a.'s 75 to 81) was chosen for the design of oligonucleotide probe pool YC-1. Since there is no preferred codon usage in vaccinia virus in the region coding for YTGI (a.a.'s 75 to 78), all possible permutations were included in the strategy, whereas in the region coding for RCQ (a.a.'s 79 to 81), only high-frequency codons were chosen. YC-1 therefore is a mixture of 21 -mers and is 128 -fold degenerate. The polarity of YC-1 (shown at bottom) was chosen (i) to facilitate preliminary confirmation of putative positive clones by dideoxy-sequencing, using YC-1 as the primer, and (ii) to identify putative mRNA species. The standard one-letter a.a. abbreviations are used (14) 
(i)

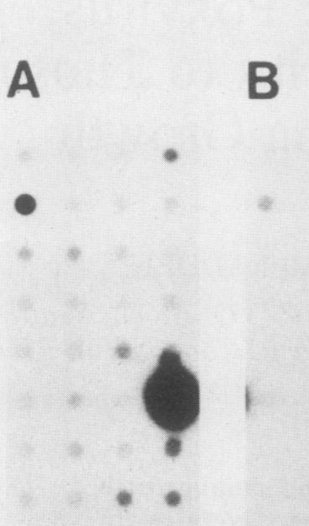

C

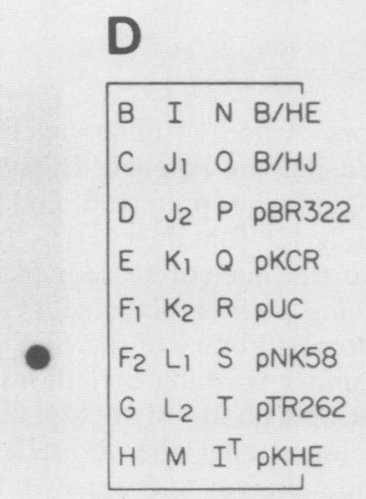

\section{(ii)}

A

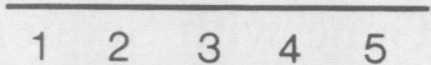

B

$\begin{array}{lllll}1 & 2 & 3 & 4 & 5\end{array}$

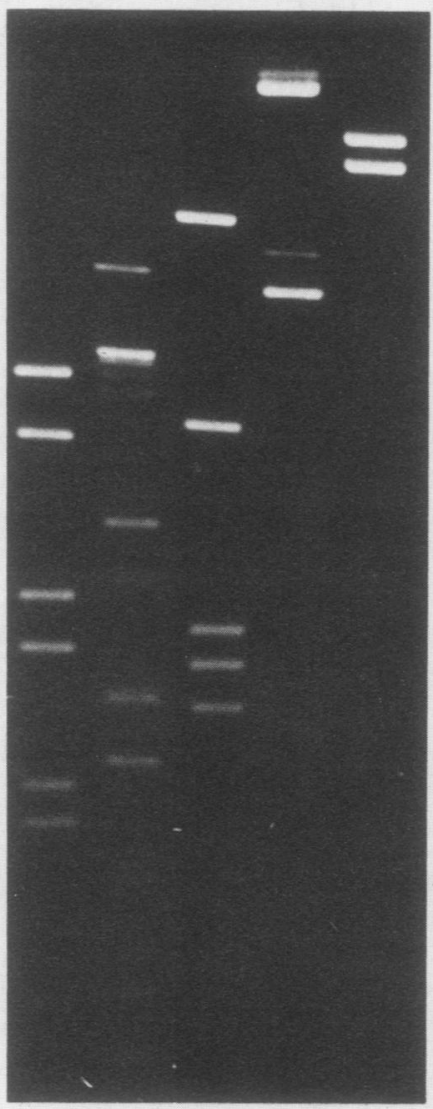

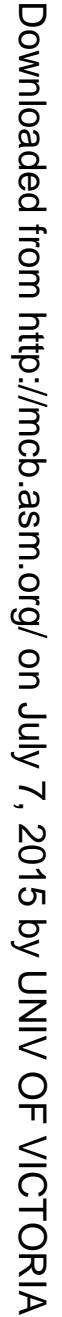

(iii)

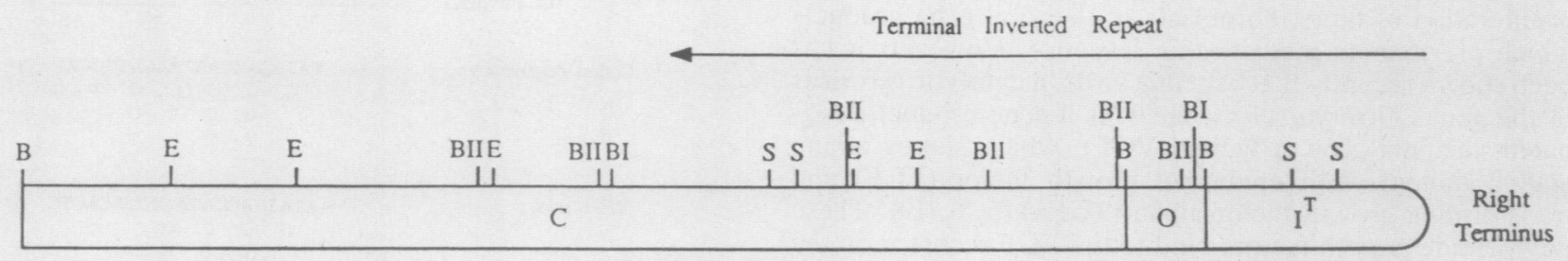

$\mathrm{KB}$

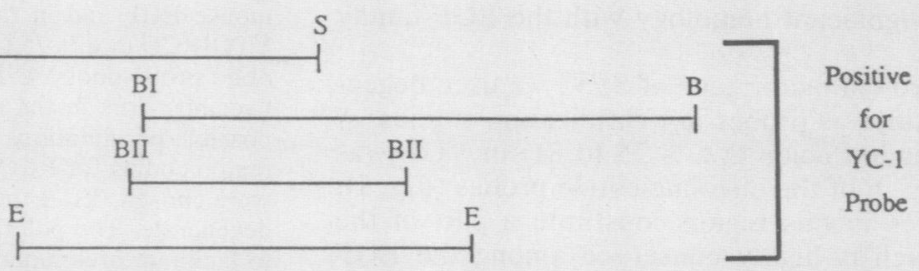

Growth factor gene 
the YC-1 probe. To further localize this region of homology, the BamHI C fragment of SFV DNA was digested with ClaI, EcoRI, BglII, SmaI, and BglI and the ability of these subfragments to hybridize with probe YC-1 was tested (Fig. 2ii). The map positions of the positive fragments (12 kilobases [kb], SmaI-BamHI; 8.5 kb, Bgll-BamHI; 4.3 kb, $B g I I I$; and $6.8 \mathrm{~kb}, E c o$ RI) are diagramed in Fig. 2iii. Results from this experiment indicate that the homology was located within a 2.3-kb region between the restriction sites for $B g l I$ at $13.5 \mathrm{~kb}$ and $S m a \mathrm{I}$ at $10.8 \mathrm{~kb}$ on the SFV map $(4,8,10)$. Since the probe YC-1 did not hybridize to BamHI fragments $\mathrm{I}^{\mathrm{T}}, \mathrm{O}$, and $\mathrm{E}$ which contain the SFV terminal inverted repeat (TIR) sequences plus unique internal sequences from the left end of the SFV genome (10), this placed the SFV growth factor (SFGF) gene within the internal unique sequences at the right end of the SFV genome, between the BglI site and the junction with the viral TIR (Fig. 2iii). This is in contrast with the location of the VGF gene, which was within the TIR of the vaccinia genome.

To further characterize this region of the SFV genome which was homologous to the VGF gene, we subcloned the 2.3-kb $B g I I-S m a I$ fragment into M13, mp18, and mp19 and determined the nucleotide sequence from the $B g I$ site to the boundary between the viral TIR and the unique internal sequence (Fig. 3), using exonuclease III-derived deletions as templates by the method of Sanger (4, 28, 29). Computer analysis revealed a single complete open reading frame capable of encoding a growth-factor-like polypeptide which is $\mathbf{8 0}$ a.a.'s in length. In addition, the sequence data revealed a portion of open reading frame T9-R, a recently described SFV gene which maps almost entirely in the viral TIR except for the N-terminal 50 a.a.'s, which are encoded in the unique internal sequences at the right end of the viral genome (29). This result confirmed the proximity of the SFGF gene to the unique internal sequence-TIR sequence junction as had been indicated by the Southern blot analysis.

The SFGF gene is quite $\mathrm{A}+\mathrm{T}$ rich $(64 \%)$, a common feature of many poxvirus genes. The DNA sequence AATATAAA upstream of the presumptive initiating ATG codon for both SFGF and open reading frame T9-R (Fig. 3) is also present upstream of VGF and several other SFV genes (29). The role of this conserved region in the expression of the SFGF gene in SFV is currently under investigation. By Northern blot analysis we detected SFGF expression as early as $2 \mathrm{~h}$ after SFV infection of CV-1 cells (data not shown). This indicates that the SFGF may be expressed as an early gene product during SFV infection, as is VGF during vaccinia infection.
The location of the SFGF gene within the unique SFV sequences adjacent to the right viral TIR is highly significant. A recently discovered tumorigenic leporipoxvirus, malignant rabbit fibroma virus (MRV), has been shown to be a recombinant between myxoma virus, the agent of myxomatosis, and SFV (4). MRV is of particular interest because it induces fibromas in infected rabbits that at early periods are indistinguishable from those induced by SFV. Mapping and sequencing studies $(4,29)$ indicate that only 5 to $7 \mathrm{~kb}$ of sequences derived in large part from the SFV TIR region have been transferred by genetic recombination into a myxoma virus genetic background to generate the recombinant MRV genome. Since this stretch of SFV DNA transferred to MRV includes an intact SFGF gene (C. Upton, J. Macen, and G. McFadden, manuscript in preparation), the putative SFGF protein would clearly be a candidate mediator of tumorigenicity for both MRV and SFV.

The deduced sequence of SFGF was compared with that of EGF, TGF $\alpha$, and VGF (Fig. 4). Of the 13 conserved tyrosine, cysteine, glycine, and arginine residues characteristic of this growth factor family, 12 residues were retained in the SFGF sequence. More importantly, the six cysteine residues which form the three disulfide bridges critical for proper folding in EGF and TGF $\alpha$ were all conserved in SFGF. Only one of the cysteine loops of SFGF (13 residues, from a.a.'s 47 to 61 ) was different in size from the corresponding loop of EGF, TGF $\alpha$, and VGF, which are all 10 a.a.'s in length. In the highly conserved stretch from a.a.'s 33 to 77 of SFGF, more than $50 \%$ of the a.a. residues in the SFV sequence had an identical counterpart in at least one of the other three growth factors. Perhaps the most striking feature of the SFGF sequence was the lack of any obvious hydrophobic cluster at the $\mathrm{C}$-terminus which could direct the putative polypeptide to a membrane-associated location. In fact, the entire 80 -a.a. SFGF is very similar in size to the secreted form of the VGF precursor, which cleaves at residues 20 and 96 to yield a 77 -a.a. extracellular polypeptide (25). At the N-terminal end of the SFGF gene there is a hydrophobic sequence from a.a.'s 6 to 17 which is reminiscent of the signal sequence of secreted proteins. However, it remains to be determined whether SFGF is cleaved and secreted like its vaccinia virus counterpart. Inspection of all open reading frames both upstream and downstream from the SFGF gene did not reveal any obvious sequence homologies to the precursor sequences cleaved from EGF, TGF $\alpha$, or VGF proteins before secretion. Thus, the evolutionary origin of SFGF with respect to these growth factors cannot as yet be ascertained.

FIG. 2. Identification and mapping of the SFV growth factor gene. Panel (i): Dot blot analysis. The cloned SFV BamHI fragments (B to $\mathrm{T})$ and the subclones of $B a m \mathrm{HI}$ fragment $\mathrm{A}(\mathrm{pKB} / \mathrm{HE}, \mathrm{pKB} / \mathrm{HJ}$, and $\mathrm{pKHE})$, as well as the cloning vectors used have been previously described $(10,30)$. The positive control pNK-58 contains the 1.4-kb HincII fragment of vaccinia strain WR which encodes the entire VGF gene cloned into the HincII site of pUC13. In (A) approximately $10 \mathrm{ng}$ of plasmid DNA of each clone, subclone, or vector was denatured, bound to nitrocellulose filters, and hybridized with $5 \times 10^{6} \mathrm{cpm}$ of ${ }^{32} \mathrm{P}$-end-labeled YC-1 probe per ml (specific activity, $10^{9} \mathrm{cpm} / \mu \mathrm{g}$ of DNA). In iB and iC, $2 \mathrm{ng}$ and $0.4 \mathrm{ng}$ of each plasmid DNA, respectively, were tested. In panel D is shown the code for the SFV BamHI fragments (B to T), the SFV terminal fragment $\left(\mathrm{I}^{\mathrm{T}}\right)$, the three subclones of SFV BamHI fragment A $(\mathrm{B} / \mathrm{HE}, \mathrm{B} / \mathrm{HJ}$, and pKHE), the four cloning vectors (pBR322, pKCR, pUC, and pTR262), and the positive control (pNK58). Panel (ii): Southern blot analysis. The purified BamHI C fragment of SFV was digested with ClaI (lane 1), EcoRI (lane 2), BglII (lane 3), SmaI (lane 4), or Bgll (lane 5), blotted, and hybridized with a [32P]YC-1 probe. Size markers (in kilobases) are from $\lambda$ DNA digested with EcoRI and HindIII. The 6.8-kb EcoRI fragment (panel A, lane 2) is only faintly positive in the photograph (B) but clearly visible in the original autoradiogram. Panel (iii): Map of the SFV right terminus. The three terminal Bam HI fragments of the SFV right terminus $\left(C, O\right.$, and $\left.I^{T}\right)$ which includes the entire 12.4-kb right TIR sequence, are diagrammed. The locations of the BamHI (B), EcoRI (E), BgII (BI), BgIII (BII), and SmaI (S) sites are indicated (4, 10). Only the ClaI sites in the vicinity of the growth factor were mapped and are not shown, but their positions are consistent with the data in panel ii, lane 1 . Fragments which hybridized to the YC-1 probe (iiB) are diagrammed, and the site of the deduced SFGF gene (Fig. 3) is indicated at the bottom. 
Bgl I

1

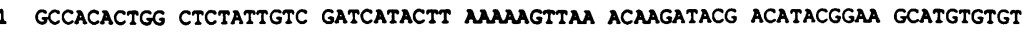

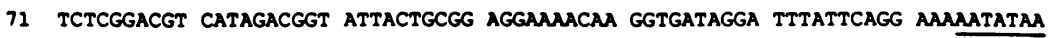

HIncII

I

141 atatatacg acatactata ataAacgtag tanactgcca gtatatctgt caActgcat ggttgcGacc

211 CTTATtGTt ATGGCGTAAT AAA ATG GCG ACG CGg AAC CTA GTG GCC TCT CTA TTA MET Ala Thr Arg Asn Leu Val Ala Ser Leu Leu

264 TGT ATt ATG TAC GCG GTA CAC GCG ATG AAC GAT TAT CTG TAT ATt GTC AAA CAT Cys Ile Met Tyr Ala Val his Ala Met Asn Asp Tyr Leu Tyr Ile Val Lys His

321 GTT AAA GTA TGT AAT CAC GAC TAT GM MAC TAT TGT CTG AAT AAC GGA ACT TGT Val Lys Val Cys Asn h1s Asp Tyr Glu Asn Tyr Cys Leu Asn Asn Gly thr Cys

ClaI

I

375 TTT ACT ATA GCA TTA GAC AAT GTA TCG ATT ACC CCA TTT TGT GTA TGT CGT ATT Phe Thr Ile Ala Leu Asp Asn Val Ser Ile Thr Pro phe Cys val Cys Arg Ile

429 AaC TAC GAG GGA AGT AGA TGT CAG TTC ATT AAT TTA GTT ACT TAT TAA GTGATAACCA Asn Tyr Glu Gly Ser Arg Cys Gln phe Ile Asn Leu Val Thr Tyr

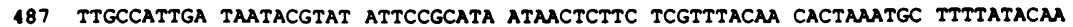

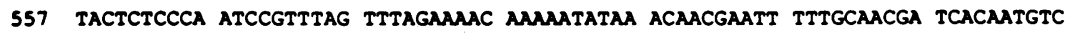

627 GCGTACTTTA TTGAGATTT TGGAagATG TGCCATGAGC GACGTGACGA ITGTCGTAGg AAACTTAAG

697 TTITTTGCAC ATAAACTAAT TTTATCTCTT CACTCGGACT ACTTTTACCG ITTGTTTAAC GGTGGTTTTA

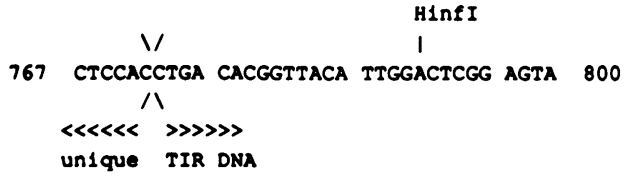

FIG. 3. Deduced a.a. sequence of the SFGF. The DNA sequence from the SFV BglI site at nucleotide 13,169 (designated nucleotide 1 here) from the right viral terminus to the TIR junction at nucleotide 12,397 was determined. The nomenclature for SFV sequences from the terminus is described elsewhere (28). The position of the initiating ATG codon for the T9-R open reading frame, which extends from the unique internal sequences into the right TIR is indicated (29). The conserved AATATAAA sequence upstream of each initiating ATG is underlined. MET, initiating methionine. 

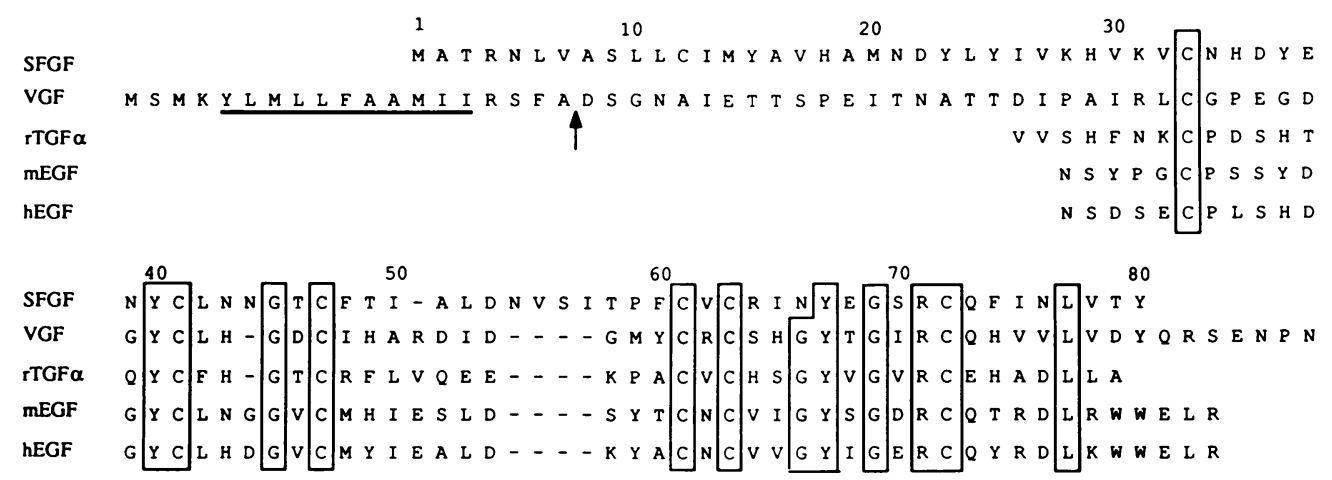

VGF

TTSYIPSPGIMLVLVGIIIITCCLLSVYRFTRRTKLPIQDMVVP

FIG. 4. Comparison of the SFGF a.a. sequence with other members of the EGF-TGF $\alpha$ family. The 80-a.a. sequence of the SFGF sequence is compared with the vaccinia strain WR VGF precursor, and the secreted peptides in rat TGF $\alpha$ (rTGF $\alpha$ ), mouse EGF (mEGF), and human EGF (hEGF) $(5,6)$. Identical residues are indicated in blocks. The proposed N-terminal signal sequence and hydrophobic C-terminal membrane spanning site of the VGF are underlined (6), and the deduced cleavage sites for the generation of the secreted polypeptide derived from the VGF precursor (25) are indicated by arrows.

The role of EGF- TGF $\alpha$-like growth factors in the poxvirus life cycle is still unclear (24). VGF purified from the supernatant of vaccinia virus-infected cells binds to EGF receptors and can stimulate the tyrosine kinase activity of these receptors in a manner analogous to that induced by EGF or TGF $x(16,27)$. Our results indicate that the presence of a growth-factor-like gene may be a common feature of the poxvirus family. The question of whether the characteristic proliferative responses induced by the tumorigenic poxviruses such as SFV are in fact mediated by this growth factor may now be addressed directly by in vitro mutagenesis in this region of the viral genome.

C.U. is a postdoctoral fellow and G.M. is a scholar of the Alberta Heritage Foundation for Medical Research. W.C. is supported by Oncogen. This work was supported in part by operating grants (G.M.) from the Alberta Cancer Board and the National Cancer Institute of Canada. The computer resource BIONET (Intelligenetics, Inc.) used for some of the sequence management was funded by a Public Health Service grant from the National Institutes of Health.

We thank Lydia Wizental for making the oligonucleotides used in this study, Tim Rose for helping with the sequencing, Adrian Wills and Robert Maranchuk for technical assistance, and Beverly Bellamy for help with the manuscript.

\section{LITERATURE CITED}

1. Allison, A. C. 1966. Immune responses to SFV in adult and newborn rabbits. J. Natl. Cancer Inst. 36:869-876.

2. Allison, A. C., and R. M. Friedman. 1966. Effects of immunosuppressants on Shope fibroma virus. J. Natl. Cancer Inst. 35:859-868.

3. Bearcroft, W. C. G., and M. F. Jamieson. 1958. An outbreak of subcutaneous tumors in rhesus monkeys. Nature (London) 182:195-196.

4. Block, W., C. Upton, and G. McFadden. 1985. Tumorigenic poxviruses: genomic organization of malignant rabbit virus, a recombinant between Shope fibroma virus and myxoma virus. Virology 140:113-124.

5. Blomquist, M. C., L. T. Hunt, and W. C. Barker. 1984. Vaccinia virus 19-kilodalton protein: relationship to several mammalian proteins, including two growth factors. Proc. Natl. Acad. Sci. USA 81:7363-7367.

6. Brown, J. P., D. R. Twardzik, H. Marquardt, and G. J. Todaro. 1985. Vaccinia virus encodes a polypeptide homologous to epidermal growth factor and transforming growth factor. Nature
(London) 313:491-492.

7. Brown, S. T., J. F. Nalley, and S. J. Kraus. 1981. Molluscum contagiosum. Sex. Transm. Dis. 8:227-234.

8. Cabirac, G. F., D. S. Strayer, S. Sell, and J. L. Leibowitz. 1985. Characterization, molecular cloning, and physical mapping of the Shope fibroma virus genome. Virology 143:163-170.

9. Dales, S., and B. G. T. Pogo. 1982. The biology of poxviruses. Springer-Verlag, New York.

10. Delange, A. M., C. Macaulay, W. Block, T. Mueller, and G. McFadden. 1984. Tumorigenic poxviruses: construction of the composite physical map of the Shope fibroma virus genome. J. Virol. 50:408-416.

11. Febvre, H. 1962. The Shope fibroma virus of rabbits, p. 79-111. In A. J. Dalton and F. Haguenau (ed.), Tumors induced by viruses: ultrastructural studies. Academic Press, Inc., New York.

12. Goustin, A. S., E. B. Leof, G. D. Shipley, and H. L. Moses. 1986. Growth factors and cancer. Cancer Res. 46:1015-1029.

13. Heldin, C. H., and B. Westermark. 1984. Growth factors: mechanism of action and relation to oncogenes. Cell 37:9-20.

14. IUPAC-IUB Commission on Biochemical Nomenclature. 1968. IUPAC-IUB Commission on biochemical nomenclature. A oneletter notation for amino acid sequences. Tentative rules. J. Biol. Chem. 243:3557-3559.

15. James, R., and R. A. Bradshaw. 1984. Polypeptide growth factors. Annu. Rev. Biochem. 53:259-292.

16. King, C. S., J. A. Cooper, B. Moss, and D. R. Twardzik. 1986. Vaccinia virus growth factor stimulates tyrosine protein kinase activity of A431 cell epidermal growth factor receptors. Mol. Cell. Biol. 6:332-336.

17. Massagué, J. 1985. The transforming growth factors. Trends Biochem. Sci. 10:237-240.

18. Moss, B. 1985. Replication of poxviruses, p. 685-703. In B. N. Fields (ed.), Virology. Raven Press, Publishers, New York.

19. Reisner, A. H. 1985. Similarity between the vaccinia virus $19 \mathrm{~K}$ early protein and epidermal growth factor. Nature (London) 313:801-803.

20. Roberts, A. B., and M. B. Sporn. 1985. Transforming growth factors. Cancer Surv. 4:683-705.

21. Sell, S., and C. B. Scott. 1981. An immunohistological study of Shope fibroma virus in rabbits; tumor rejection by cellular reaction in adults and progressive systemic reticuloendothelial infection in neonates. J. Natl. Cancer Inst. 66:363-373.

22. Shope, R. E. 1932. A transmissible tumor-like condition in rabbits. J. Exp. Med. 56:793-802.

23. Smith, J. W., S. S. Tevethia, B. M. Levy, and W. E. Rawls. 1973. 
Comparative studies on host responses to Shope fibroma virus in adult and newborn rabbits. J. Natl. Cancer Inst. 50:15291539.

24. Spriggs, D. R. 1986. Vaccinia virus growth factor: a surprising catch. J. Infect. Dis. 153:382-383.

25. Stroobant, P., A. P. Rice, W. J. Gullick, D. J. Cheng, I. M. Kerr, and M. D. Waterfield. 1985. Purification and characterization of vaccinia virus growth factor. Cell 42:383-393.

26. Tompkins, W. A. F., R. M. Schultz, and G. V. S. V. Rama Rao. 1973. Depressed cell-mediated immunity in newborn rabbits bearing fibroma virus-induced tumors. Infect. Immun. 7:613619.

27. Twardzik, D. R., J. P. Brown, J. E. Ranchalis, G. J. Todaro, and B. Moss. 1985. Vaccinia virus-infected cells release a novel polypeptide functionally related to transforming and epidermal growth factors. Proc. Natl. Acad. Sci. USA 82:5300-5304.

28. Upton, C., and G. McFadden. 1986. DNA sequence homology between the terminal inverted repeats of Shope fibroma virus and an endogenous cellular plasmid species. Mol. Cell. Biol. 6:265-276.

29. Upton, C., and G. McFadden. 1986. Tumorigenic poxviruses: analysis of viral DNA sequences implicated in the tumorigenicity of SFV and malignant rabbit virus. Virology 152:308321.

30. Wills, A., A. M. Delange, C. Gregson, C. Macaulay, and G. McFadden. 1983. Physical characterization and molecular cloning of the Shope fibroma virus DNA genome. Virology 130: $403-414$. 